\title{
Originales
}

\section{Sistema de auriculares en teleoperadores: estudio en una central de llamadas del sur de España}

\author{
Headset System in Teleoperators: Study in a Call Centre in Southern Spain
}

\section{Concepción Flores Muñoz'1, Francisco de Asís Manchado López², Antonio Ranchal Sánchez ${ }^{3}$}

1. Centro de Prevención de Riesgos Laborales de Córdoba. España.

2. Área de Vigilancia de Salud. Centro de Prevención de Riesgos Laborales de Córdoba. España.

3. Unidad de Medicina del Trabajo. Hospital Universitario Reina Sofía de Córdoba. Servicio Andaluz de Salud. Facultad de Medicina y Enfermería, Universidad de Córdoba. IMIBIC. España.

Recibido: 30-11-2018

Aceptado: 01-03-2019

\section{Correspondencia}

Antonio Ranchal Sánchez

Médico del Trabajo

Hospital Universitario Reina Sofía. Córdoba. España

Correo electrónico: antonio.ranchal.sspa@juntadeandalucia.es

\section{Resumen}

Introducción: El personal teleoperador de las centrales de llamadas telefónicas, está sometido a ruido ambiental y al nivel sonoro de los auriculares con el posible desarrollo de pérdida auditiva.

Objetivos: El objetivo principal del estudio fue evaluar si el tipo de sistema de auriculares empleado por los teleoperadores de una central de llamadas influía sobre la aparición de pérdida auditiva asimétrica.

Material y métodos: Estudio observacional descriptivo realizado en teleoperadores de una central de llamadas del sur de España. Muestreo oportunista a partir del examen de vigilancia de salud de 2018, con audiometría y cuestionario auto-cumplimentado. La variable dependiente fue la presencia de pérdida auditiva asimétrica (diferencia de umbral interaural de 15 dB o más en al menos una frecuencia). Se realizó análisis bivariante, y se usaron los programas estadísticos SPSS versión 25 y EPIDAT versión 4.2.

Resultados: La pérdida auditiva asimétrica en la muestra estudiada se relacionaba significativamente con la edad de los sujetos $(\mathrm{Z}=-3,606, \mathrm{p}<0,000)$, pero no con el tipo de sistema de auriculares utilizado ni con otras variables sociodemográficas, laborales o clínicas analizadas.

Conclusiones: El uso de auriculares del sistema monoaural no parece influir sobre la pérdida auditiva asimétrica en el personal teleoperador de la muestra estudiada.

Med Segur Trab (Internet). 2019;65(254):37-48

Palabras clave: central de llamadas, teleoperadores, pérdida auditiva unilateral, ambliaudia, vigilancia de la salud.

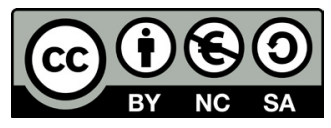

https://creativecommons.org/licenses/by-nc-sa/4.0/ 


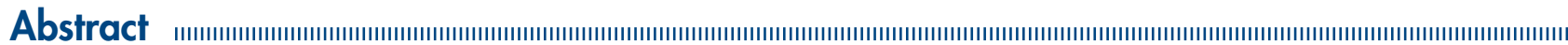

Introduction: The Call Center Phone Operator is subject to environmental noise and to the headphones volume sound with possible hearing loss effects.

Objectives: The main objective of the study was to assess whether the type of headphone system used by call center operators incidenced the asymmetrical hearing loss.

Material and methods: Descriptive observational study carried out on teleoperators of a call centre in the south of Spain. Opportunity sampling technique carried out from the health surveillance examination from 2018, with audiometry and self-completed questionnaire. The dependent variable was the presence of asymmetric hearing loss (interaural threshold value of $15 \mathrm{~dB}$ or more in at least one frequency). Bivariate analysis was performed, and statistical programs SPSS version 25 and EPIDAT version 4.2 were used.

Results: Asymmetric hearing loss in the studied sample was significantly related to the age of the subjects $(Z=-3,606, p<0,000)$, but not to the type of headphone system used or to other socio-demographic, occupational or clinical variables analysed.

Conclusions: The use of mono headphones does not seem to be related with an asymmetric hearing loss in the telephone operators of the studied sample.

Med Segur Trab (Internet). 2019;65(254):37-48

Keywords: call centre; teleoperators; hearing loss, unilateral; amblyaudia; occupational health. 


\section{INTRODUCCIÓN}

Las centrales de llamadas telefónicas son un sector de actividad en auge. En 2016 supuso un volumen de negocio global de 1.723,96 millones de euros y dio empleo a 70.525 personas en España ${ }^{1}$. Dentro del sector servicios están encuadrados en el grupo de actividades administrativas y servicios auxiliares (N) de la Clasificación Nacional de Actividades Económicas (CNAE) con el código 8220 (actividades de las centrales de llamadas $)^{2}$. Además, interacciona con numerosos servicios como: aseguradoras, servicios públicos, finanzas, telecomunicaciones, venta por correspondencia, servicios post-venta, agencias de viajes, etc $^{3}$.

Son centros que actúan de interfaz entre empresas (o administraciones) y sus clientes mediante la gestión de llamadas telefónicas entrantes y/o salientes. Bajo una misma denominación se reagrupan una gran variedad de actividades (asistencia jurídica y técnica, información, telemarketing, gestión comercial,...). Pueden pertenecer a la propia empresa o encontrarse externalizados. Sus acciones pueden ser puntuales o mantenidas en el tiempo. Tienen unas características propias, entre ellas: el acoplamiento de telefonía e informática, la organización específica del trabajo con actuaciones bien definidas, el importante control jerárquico y una constante evaluación de la actividad y rendimiento del trabajador ${ }^{4}$.

El personal teleoperador de estas empresas está sometido a una exposición sonora compleja derivada, por un lado, de la presencia de ruido ambiental y por otra, del nivel sonoro originado en los auriculares. El ruido ambiente puede condicionar la inteligibilidad de las conversaciones y, por tanto, tener influencia sobre la regulación del nivel sonoro del auricular 5 . De modo que un ruido de fondo elevado puede hacer que el teleoperador aumente el volumen de recepción del auricular y tienda a hablar más fuerte, aumentando a su vez el ruido ambiente ${ }^{6}$. Al respecto, existen distintos tipos de auriculares según la posición respecto al oído: circumaurales (rodean completamente la oreja), supraaurales (los altavoces cubren la mayor parte del pabellón auditivo), intraaurales o intraauriculares (se introducen dentro del canal auditivo), y de conducción ósea (se sitúan en la superficie del cráneo) ${ }^{7}$. Pueden además diferenciarse entre monoaurales (constan de un auricular) o binaurales (dos auriculares).

Revisando la literatura, varios estudios han medido los niveles de ruido emitidos por los auriculares así como el ruido de fondo que existe en los lugares de trabajo ${ }^{8,9,10}$, llegando a una conclusión unánime: el nivel diario de exposición a ruido de los teleoperadores de las centrales de llamadas es poco probable, aunque posible, que esté por encima de los valores superior e inferior que dan lugar a una acción recogidos en la actual legislación ${ }^{11}$. El Real Decreto 286/2006 ${ }^{11}$, que traspone la Directiva 2003/10/CE ${ }^{12}$, determina en su artículo 5 los valores límite de exposición y los valores de exposición que dan lugar a una acción, referidos a los niveles de exposición diaria y a los niveles de pico. Dichos valores límite de exposición tienen en cuenta la atenuación que procuran los protectores auditivos individuales utilizados por los trabajadores siendo las cifras de 87 dB para niveles de exposición diaria y $140 \mathrm{~dB}$ en relación a niveles pico. Los valores superiores de exposición que dan lugar a una acción son de 85 y 137 dB, y los inferiores de 80 y $135 \mathrm{~dB}$, respectivamente.

Son escasos los artículos científicos recientes que evalúan específicamente el ruido y/o sus efectos en la audición de los trabajadores de centrales de llamadas ${ }^{13,14,15,16}$. Beyan $2016^{13}$, presenta el caso clínico de un operador masculino de 30 años de una central de llamadas domiciliaria que sufre pérdida de audición inducida por ruido. D'Alcamo $2011^{14}$ en Italia y Chi CF $^{15} 2008$ en Taiwan, hacen una valoración subjetiva del ruido o los síntomas auditivos, mediante cuestionarios autoadministrados. El ruido (ambiental o auricular) fue juzgado de manera negativa o parcialmente negativa por el $52 \%$ de los empleados. El malestar en los oídos podría atribuirse al uso inevitable de un teléfono o auriculares más el ruido de fondo. Venet $2018^{16}$, estudia la fatiga auditiva de 55 teleoperadores dividiéndolos en dos grupos (39 operadores que trabajaron con auriculares 
en una central de llamadas, y un grupo control de 16 participantes que realizaron tareas administrativas o de gestión). La fatiga auditiva descrita por los trabajadores no se reflejó con diferencias significativas en pruebas objetivas de fatiga auditiva central o periférica: audiometría de tonos puros y productos de distorsión de otoemisiones acústicas. La sensación de fatiga podría estar causada por fatiga cognitiva. La exposición de ruido a través del auricular ponderada a 8 horas $(65,7 \mathrm{~dB})$ estaba considerablemente por debajo de los niveles de acción definidos por la legislación vigente y comparable al grupo control que realizó tareas administrativas $(65,3 \mathrm{~dB})$.

Sin embargo, ningún estudio ha evaluado el posible desarrollo de pérdida auditiva asimétrica en teleoperadores por el hecho de utilizar auriculares con sistema monoaural. En este sentido el objetivo principal del estudio fue evaluar si el tipo de sistema de auriculares empleado por los teleoperadores de una central de llamadas de la provincia de Málaga influía sobre la aparición de pérdida auditiva asimétrica.

Como objetivo secundario nos planteamos relacionar la posible pérdida auditiva asimétrica con factores de tipo sociodemográfico, laborales y con antecedentes médicos.

\section{MATERIAL Y MÉTODOS}

\section{Diseño epidemiológico y variables recogidas}

Se realizó un estudio observacional, descriptivo, a partir de los exámenes de la vigilancia de la salud en una empresa privada del sector. La variable dependiente fue la presencia de pérdida auditiva asimétrica, considerándose como tal, una diferencia de umbral interaural de $15 \mathrm{~dB}$ o más en al menos una frecuencia.

Las variables independientes tenidas en cuenta fueron de tipo demográficas, antropométricas, clínicas y laborales según se muestra en el siguiente cuadro-resumen.

\begin{tabular}{|c|c|c|c|}
\hline Demográficas & Antropométricas & Clínicas & Laborales \\
\hline $\begin{array}{l}\text { Sexo (hombre, } \\
\text { mujer) }\end{array}$ & $\begin{array}{l}\text { Peso (kg) } \\
\text { Talla (m) } \\
\text { IMC (Peso/Talla²) } \\
\text { Perímetro } \\
\text { abdominal (cm) }\end{array}$ & $\begin{array}{l}\text { - Antecedentes familiares } \\
\text { de sordera antes de los } \\
60 \text { años } \\
\text { - Consumo de tabaco } \\
\text { - Consumo de alcohol } \\
\text { - Hipertensión arterial } \\
\text { - Diabetes mellitus } \\
\text { - Infecciones de oído de } \\
\text { repetición o drenajes } \\
\text { timpánicos en la infancia } \\
\text { - Fármacos ototóxicos (1) } \\
\text { - Suma ponderada de } \\
\text { ruido percibido (2) }\end{array}$ & $\begin{array}{l}\text { - Tipo de auricular: monoaural (uso } \\
\text { fijo derecho, uso fijo izquierdo, uso } \\
\text { compartido) o binaural } \\
\text { - Horas semanales de atención } \\
\text { a llamadas (tiempo efectivo de } \\
\text { atención a llamadas durante la } \\
\text { semana) } \\
\text { - Antigüedad en el puesto de trabajo } \\
\text { (en meses) } \\
\text { - Pausas de trabajo (ninguna, horaria, } \\
\text { otra) } \\
\text { - Reciclaje formativo en el uso y } \\
\text { mantenimiento del equipo (ninguno, } \\
\text { anual, bianual, otro) } \\
\text { - Limitador de choque acústico } \\
\text { (presente, ausente, desconocido) } \\
\text { - Volumen del auricular (necesidad de } \\
\text { elevar el volumen del auricular al } \\
\text { máximo) }\end{array}$ \\
\hline
\end{tabular}

1. Antibióticos intravenosos, macrólidos, salicilatos, $\beta$-bloqueantes, diuréticos

2. Obtenida de los datos del cuestionario modificado de hábitos cotidianos y ruido ambiental, mediante la siguiente fórmula: $\mathrm{A}+\mathrm{B}+\mathrm{C}+\mathrm{D}+(\mathrm{E} \times \mathrm{x}$ horas semanales / 5) + (auriculares $)+(\mathrm{F} 1 \mathrm{x}$ horas semanales $)+(\mathrm{F} 2 \mathrm{x}$ horas semanales $)+(\mathrm{F} 3 \mathrm{x}$ horas semanales $)+(\mathrm{G} \times \text { días al año/7 })^{17,18}$, donde 


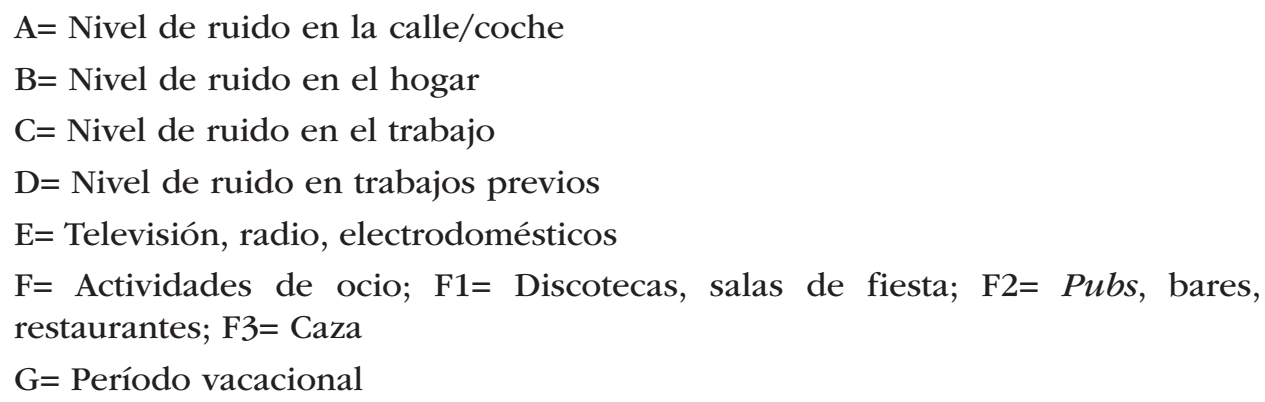

\section{Población de estudio}

La población de estudio fueron teleoperadores de una central de trabajo privado de llamadas en Málaga. La muestra se obtuvo mediante muestreo no probabilístico del personal que, cumpliendo los criterios de inclusión, aceptó participar en el estudio aprovechando el examen de vigilancia de la salud realizado en 2018, obteniéndose una muestra de 55 teleoperadores y teleoperadoras.

Una vez realizado el protocolo pertinente de investigación y obtenida la autorización correspondiente por parte del Comité de Ética de la Investigación (CEI) de Córdoba, se procedió a recopilar datos de la historia clínico-laboral de los trabajadores (demográficos, clínicos, laborales, de pruebas complementarias) mediante una plantilla o checklist, así como los resultados de la audiometría del examen de vigilancia de salud realizado. Dicho reconocimiento de vigilancia de salud de los trabajadores se realizó durante el mes de marzo de 2018. Se citó a los trabajadores en horario de consulta para completar el cuestionario de hábitos cotidianos y ruido ambiental ${ }^{17}$, así como para completar aspectos que no estuvieran disponibles en los archivos del trabajador. Los datos fueron trasladados a una base de datos anonimizada utilizando el programa Excel para su tratamiento estadístico posterior.

\section{Métodos estadísticos}

Para el estudio inferencial y contraste de hipótesis se procedió a un análisis bivariante entre la variable dependiente y las variables independientes mencionadas previamente, haciendo uso del test de $\chi^{2}$ o chi-cuadrado en caso de variables cualitativas y la t de student o U de Mann-Whitney en caso de variables cuantitativas, una vez comprobada o descartada normalidad, respectivamente, mediante la prueba de Kolmogorov-Smirnov. Para todos los test empleados la significación estadística se fijó en p < 0,05 (2 colas).

\section{Criterios de inclusión y exclusión}

Los criterios de inclusión tenidos en cuenta en la selección de la muestra fueron: que el personal teleoperador tuviese realizada su vigilancia de salud en 2018; que la audiometría del examen de salud inicial fuese simétrica (entendida como la ausencia de una diferencia interaural mayor de $15 \mathrm{~dB}$ en alguna de las frecuencias), que al menos 15 horas semanales de trabajo de dicho personal fuese dedicado a llamadas, y que tuvieran más de 6 meses de antigüedad en el puesto de trabajo.

Como criterios de exclusión se consideraron: padecer de cofosis unilateral previa y la presencia de alteraciones unilaterales de oído externo en la otoscopia realizada durante el examen de vigilancia de salud. Éstas fueron: tapón de cerumen completo, perforación timpánica, exóstosis importante, deformidad con estrechamiento de conducto auditivo externo (CAE).

\section{Tratamiento de los datos}

Para el análisis estadístico de los datos se utilizó el programa Statistical Package for the Social Sciences (SPSS) versión 25, así como el programa de análisis epidemiológico de datos EPIDAT, versión 4.2. 


\section{Procedimientos y normás éticas seguidas}

Los datos fueron obtenidos con carácter retrospectivo una vez conseguida la aceptación del estudio por el CEI del Hospital Universitario Reina Sofía de Córdoba, cumpliéndose las normas éticas contenidas en la declaración de Helsinki (http://www. wma.net/s/index.html). Todos los participantes firmaron el obligado consentimiento informado.

Para minimizar los sesgos se realizó un pilotaje previo usando los datos de la vigilancia de salud de los teleoperadores de esta única empresa para comprobar que todo el personal estuviera sometido a niveles de ruido ambiente y medidas de prevención de riesgo específicas similares evitando factores de confusión que pudieran influir en los resultados.

\section{RESULTADOS}

Un 38\% del total del personal de la empresa a quien se realizó la vigilancia de la salud aceptó participar. De estos 90 teleoperadores, 55 cumplieron los criterios de inclusión. Del personal excluido, 21 no tenían audiometría inicial, 5 tenían asimetría en la audiometría inicial, 7 dedicaban menos de 15 horas semanales a llamadas, uno fue excluido por tapón de cerumen en la otoscopia, y otro por estrechez en el CAE.

Respecto al estudio descriptivo, la tabla I ofrece las características de la muestra de estudio. Estaba formada por 36 hombres (65,5\%) y 19 mujeres (34,5\%), con edades comprendidas entre 26 y 55 años, con una edad media de 37 años. Los datos antropométricos de dicha muestra pueden observarse también en la tabla I. Teniendo en cuenta el valor de Índice de Masa Corporal (IMC), la mayoría se encontraban en la categoría de normopeso, 14 individuos presentaron sobrepeso (25,5\%), 4 tenían obesidad grado I $(7,3 \%)$ y 2 , obesidad grado II (3,6\%). En relación al perímetro abdominal, 9 cumplían criterios de obesidad abdominal (16,4\%): 4 hombres y 5 mujeres. En cuanto a los hábitos de salud, destaca el 67,3\% que admitieron un consumo de alcohol (37 personas). Solo un $10,9 \%$ eran fumadores (6 individuos). Respecto a patología otológica, un trabajador respondió haber tenido infecciones de repetición o drenaje de oídos en su infancia y también uno, refería antecedentes familiares de sordera antes de los 60 años. Ninguno tuvo prescrita medicación ototóxica.

Tabla I. Características de la población.

\begin{tabular}{l|c|c|c|c|c|c}
\hline & Frecuencia & Porcentaje & Media & Mínimo & Máximo & DT \\
\hline Edad en Años & & & 37 & 26 & 55 & 7,36 \\
\hline Sexo & & & & & & \\
\hline Hombre & 36 & 65,5 & & & & \\
\hline Mujer & 19 & 34,5 & & & & \\
\hline IMC & & & 24,53 & 17,36 & 35,79 & 4,04 \\
\hline Estado Nutricional & & & & & & \\
\hline Bajo Peso & 1 & 1,8 & & & & \\
\hline Normopeso & 33 & 60,0 & & & & \\
\hline Sobrepeso & 15 & 27,3 & & & & \\
\hline Obesidad grado I & 4 & 7,3 & & & & \\
\hline Obesidad grado II & 2 & 3,6 & & & & \\
\hline Obesidad Abdominal & 9 & 16,4 & & & & \\
\hline Hombre & 4 & 7,3 & & & & \\
\hline Mujer & 5 & 9,1 & & & & \\
\hline
\end{tabular}

DT: Desviación Típica, IMC: Índice de Masa Corporal. 
Analizando las respuestas del cuestionario modificado de hábitos cotidianos y ruido ambiental y calculando la suma ponderada de ruido percibido (tal como se ha especificado anteriormente), se obtuvieron valores que oscilaron entre 16 y 51 puntos, siendo los intervalos de 20-30 y 30-40 los que asumieron mayor número de trabajadores, 25 y 13 individuos respectivamente.

La tabla II muestra antecedentes laborales y datos relativos al sistema de auriculares (tiempo de antigüedad en la empresa, pausas en el trabajo). El 76,4\% de los trabajadores usaban un sistema de dos auriculares; un 16,4\% un sistema monoaural en su oído derecho y $7,3 \%$ un sistema monoaural, alternando su uso entre ambos oídos. La mayoría, llevaba en la empresa más de un año (85,5\%). Todos admitieron realizar pausas en su trabajo, un $36,4 \%$ hacían descansos cada hora. El 98,2\% de la muestra negaron realizar reciclaje en el manejo y mantenimiento de sus equipos, sólo un individuo refirió realizar el reciclaje con carácter bianual. La práctica totalidad, un 98,2\% desconocía la existencia de limitador de choque acústico en su equipo de trabajo. El 67,3\% de los trabajadores negaron el uso de su auricular al volumen máximo, aunque un $29,1 \%$ y un 3,6\% contestaron que utilizaban el equipo a máximo de su volumen a veces o siempre, respectivamente. El contrato de trabajo era de 40 horas semanales de forma generalizada y la media de horas dedicadas a llamadas dentro de su horario laboral fue de 23,16 horas/semana.

Tabla II. Características de los auriculares.

\begin{tabular}{l|c|c}
\hline & Frecuencia & Porcentaje \\
\hline Sistema de auriculares & & \\
\hline 2 auriculares & 42 & 76,3 \\
\hline 1 auricular derecho & 9 & 16,4 \\
\hline 1 auricular alterna oídos & 4 & 7,3 \\
\hline Pausas en su trabajo & & \\
\hline Horaria & 20 & 36,4 \\
\hline Otra & 35 & 63,6 \\
\hline Reciclaje & & \\
\hline Bianual & 1 & 1,8 \\
\hline Ninguno & 54 & 98,2 \\
\hline Limitador choque acústico & & \\
\hline Sí & 1 & 1,8 \\
\hline No sabe & 54 & 98,2 \\
\hline Máximo volumen auricular & & \\
\hline No o nunca & 37 & 67,3 \\
\hline A veces & 16 & 29,1 \\
\hline Sí o siempre & 2 & 3,6 \\
\hline
\end{tabular}

La tabla III muestra los resultados del estudio bivariante. La presencia de pérdida auditiva asimétrica en los sujetos de la muestra analizada mostró relación estadísticamente significativa con la edad ( $\mathrm{p}=0.000)$, IC [9.08-30.90]). 
Tabla III. Estudio bivariante.

\begin{tabular}{l|c|c}
\hline \multicolumn{1}{c|}{ Variables } & Valor & p \\
\hline Tipo de sistema auricular & $\chi^{2}=3,090$ & 0,213 \\
\hline Sexo & $\chi^{2}=1,144$ & 0,285 \\
\hline Obesidad abdominal & $\chi^{2}=1,118$ & 0,731 \\
\hline Antecedentes familiares de sordera antes de 60 años & $\chi^{2}=4,583$ & $0,032^{*}$ \\
\hline Consumo tabaco & $\chi^{2}=1,039$ & 0,308 \\
\hline Consumo alcohol & $\chi^{2}=1,656$ & 0,198 \\
\hline Diagnóstico de hipertensión arterial & $\chi^{2}=0,226$ & 0,634 \\
\hline Infecciones o drenajes en infancia & $\chi^{2}=4,583$ & $0,032^{*}$ \\
\hline Pausas & $\chi^{2}=0,070$ & 0,792 \\
\hline Reciclaje & $\chi^{2}=4,583$ & $0,032^{*}$ \\
\hline Limitador & $\chi^{2}=4,583$ & $0,032^{*}$ \\
\hline Volumen al máximo & $\chi^{2}=2,403$ & 0,301 \\
\hline Suma ponderada de ruido percibido & $\mathrm{t}=1,622$ & 0,117 \\
\hline Índice de masa corporal & $\mathrm{t}=-0,499$ & 0,620 \\
\hline Edad & $\mathrm{Z}=-3,606$ & $0,000^{*}$ \\
\hline Antigüedad en el puesto & $\mathrm{Z}=-0,447$ & 0,655 \\
\hline
\end{tabular}

Encontramos una edad media de 45 años en aquellos teleoperadores con pérdida auditiva asimétrica respecto a 35,2 años entre los que no presentaban tal eventualidad $(\mathrm{Z}=-3,606, \mathrm{p}<0,000)$ (figura 1).

Figura 1. Media de edad en función de presencia o ausencia de pérdida auditiva asimétrica.

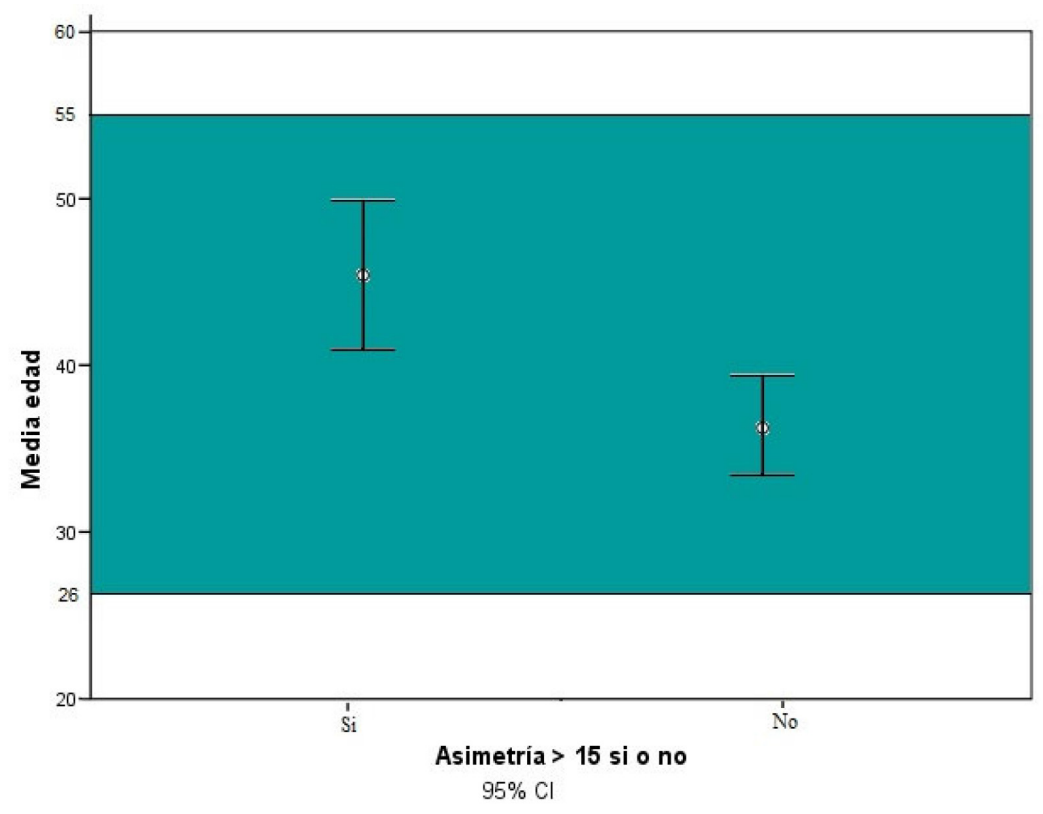

A pesar de obtener una $\mathrm{p}<0.05$ al realizar el contraste de hipótesis entre pérdida auditiva asimétrica y las variables: antecedentes familiares de sordera antes de los 60 años, antecedentes personales de infecciones de repetición o drenaje de oídos en la infancia, realizar un reciclaje formativo en el uso y mantenimiento del equipo y la presencia de limitador de choque acústico en el equipo, no se cumplieron criterios de validez para Chi-cuadrado (tabla III). 


\section{DISCUSIÓN}

El objetivo principal del estudio era evaluar si el tipo de sistema de auriculares empleado por teleoperadores de una central de llamadas influía sobre la aparición de pérdida auditiva asimétrica. Los resultados obtenidos muestran que la pérdida auditiva asimétrica en la muestra estudiada se relacionaba significativamente con la edad de los sujetos pero no con el tipo de sistema de auriculares utilizado ni con otras variables sociodemográficas, laborales o clínicas analizadas.

Otros autores también indican que la prevalencia de pérdida auditiva aumenta con la edad, pasando de un $5-10 \%$ a la edad de 40 años a aproximadamente un $80-90 \%$ a los 85 años $^{19}$. Otros factores como el tabaquismo, los cambios vasculares, la adiposidad central y la diabetes mellitus mal controlada predicen la incidencia de pérdida auditiva a los 15 años de seguimiento longitudinal de una cohorte de sujetos ${ }^{20}$. La incidencia de pérdida auditiva asimétrica en población general es solo del $1 \%^{21}$, mientras que en individuos expuestos a ruido varía ampliamente entre 4,7 y 49,2\% $\%^{22,23,24,25,26,27}$.

En nuestro estudio hemos medido dicha pérdida mediante audiometría manual (en pasos de $5 \mathrm{~dB}$ ) la cual tiene un margen de error para frecuencias individuales que varía entre $\pm 9,6$ y $\pm 14,2 \mathrm{~dB}$ (con un nivel de confianza del 95\%) ${ }^{28}$. De ahí que nos planteásemos una diferencia de umbral interaural de al menos $15 \mathrm{~dB}$ en una o más frecuencias a la hora de considerar la presencia de pérdida auditiva asimétrica. Teniendo en cuenta esta consideración, obtuvimos una pérdida auditiva asimétrica en un $18,2 \%$ de la muestra, que supera las previsiones para población general no expuesta a ruido $^{21}$. Por otro lado, en comparación con la población trabajadora expuesta a ruido, se encuentra dentro del rango descrito por Masterson et al $\left(2,4\right.$ a 22,6\% ${ }^{29}$ quienes realizaron una revisión sistemática que sólo incluyó estudios hasta 2015 con un corte de umbral asimétrico $>15 \mathrm{~dB}$ para cualquier frecuencia de 0,5 a $8 \mathrm{kHz}$; y muy inferior a la encontrada por Xiaoxiao et al $(49,2 \%)^{27}$ en 2016 con similares criterios de asimetría.

Existen dos teorías que tratan de explicar el desarrollo de asimetría durante la pérdida auditiva inducida por ruido ${ }^{26}$. La primera tiene relación con el llamado efecto "sombreado de la cabeza". Según ella, la estructura más o menos esférica de la cabeza con una disposición de un oído a cada lado de esta esfera, hace que un oído quede más protegido que otro cuando se expone a una fuente de sonido procedente de un lugar del espacio. La segunda teoría presupone una mayor susceptibilidad del oído izquierdo respecto al derecho, especialmente en el hombre, cuando el trabajador se enfrenta a una exposición perjudicial para su audición.

La susceptibilidad es un aspecto interesante en salud laboral para identificar al personal especialmente sensible. La susceptibilidad genética a la pérdida de audición inducida por ruido ha sido claramente demostrada en animales. El descubrimiento de factores genéticos humanos que predisponen a las personas a este efecto se ha visto obstaculizado fundamentalmente porque familias donde todos los sujetos estén expuestos a idénticas condiciones de ruido son casi imposibles de recopilar. Por ello, la investigación se ha enfocado al cribado de mutaciones puntuales comunes en el genoma (genes de intercambio de iones potasio, de proteínas de choque térmico, otocaterina 15, miosina 14) persiguiendo demostrar su presencia en sujetos susceptibles respecto a los resistentes a la pérdida de audición por ruido ${ }^{22}$.

También podría favorecer una especial sensibilidad determinados antecedentes patológicos. En este sentido, los lactantes con otitis media lo suficientemente grave como para causar una alteración de la transmisión de señal aferente (por ejemplo, pérdida auditiva conductiva) parecen estar particularmente en riesgo de desarrollar deficiencias auditivas centrales duraderas ${ }^{30}$. La "ambliaudia" es un término utilizado para describir la dificultad auditiva persistente experimentada por personas con un historial de pérdida auditiva asimétrica durante una ventana crítica del desarrollo del cerebro. Durante los períodos críticos del desarrollo cerebral infantil, la entrada 
auditiva desequilibrada asociada con pérdida auditiva asimétrica puede conducir a anomalías en el procesamiento binaural. Los pacientes con ambliaudia podrían padecer déficits a largo plazo en la percepción auditiva incluso con corrección o resolución de la pérdida auditiva asimétrica ${ }^{31}$. El sistema nervioso adulto puede flexibilizar y adaptar las señales binaurales de acuerdo con las demandas auditivas impuestas por el ambiente particular ${ }^{32}$.

Tanto la herencia genética como los posibles efectos a largo plazo de la ambliaudia en relación con la pérdida auditiva asimétrica ha resultado difícil de corroborar en nuestro estudio, puesto que sólo un trabajador ha tenido antecedentes familiares de sordera antes de los 60 años y otro ha admitido haber sufrido infecciones o drenajes de oído en la infancia. La vigilancia de salud respecto a una especial sensibilidad de estos sujetos obliga a un seguimiento más estrecho de su audición, utilizando, si fuese preciso, otras pruebas más sensibles, como la distorsión de las otoemisiones acústicas (Vinodh, $2010)^{33}$.

El objetivo secundario del estudio era analizar la posible pérdida auditiva asimétrica con factores de tipo sociodemográfico, laborales y con antecedentes médicos. Los resultados del estudio muestran diferencias en relación al sexo. De modo que la prevalencia de asimetría ha sido mayor en hombres, hecho que también mencionan otros autores $^{24,26,27}$. Además observamos que se ha afectado con mayor frecuencia el oído derecho respecto al izquierdo, a diferencia de los datos procedentes de la literatura ${ }^{23,24,25,26}$. Ninguna de estas diferencias ha obtenido significación estadística. Puede ser que la muestra sea insuficiente y no exista potencia estadística para ponerlas en evidencia. Para valorar el ruido autopercibido se utilizó una encuesta. El uso de datos autoinformados en diferentes actividades y situaciones de la vida cotidiana podría contener sesgos de exageración o de memoria selectiva. Sin embargo, hay estudios ${ }^{35,36}$ que demuestran que las personas pueden hacer una estimación razonable de la intensidad de los eventos y nivel general de ruido que experimentan, al existir una correlación significativa entre las estimaciones subjetivas del individuo sobre su exposición general al ruido (mediante cuestionarios) y la exposición media diaria real al ruido (medida objetivamente a través de dosimetría). En todo caso, se demandan cuestionarios adaptados y validados para la población trabajadora que midan intensidad y tiempo de exposición a fuentes de ruido de la vida cotidiana.

Las principales limitaciones de nuestro estudio han sido el tamaño muestral y el posible sesgo de selección que pueden afectar a la validez interna del estudio. Por ello sería interesante ampliarla mediante un estudio multicéntrico. Sin embargo, la mayoría de estos centros suelen ser privados y no todas las empresas están dispuestas a dar su consentimiento para recoger datos de sus trabajadores o a participar en este tipo de estudios. Al respecto, este trabajo tiene la fortaleza de haber sido realizado en una empresa privada, líder en su sector, existiendo apenas estudios en España realizados en estos centros. Además es pionero en la temática propuesta.

Para finalizar, podemos concluir que el uso de auriculares del sistema monoaural no parece influir sobre la pérdida auditiva asimétrica en el personal teleoperador de la muestra estudiada, siendo mayor la presencia de asimetría en sujetos de mayor edad. No encontramos evidencia para recomendar un sistema de auricular respecto a otro (monoaural vs binaural).

\section{CONCLUSIONES}

En relación con los objetivos planteados podemos concluir:

- El tipo de sistema de auriculares empleado por los teleoperadores de una central de llamadas de la provincia de Málaga no parece influir sobre la aparición de pérdida auditiva asimétrica. 
- Encontramos diferencias significativas en la presencia de pérdida auditiva asimétrica a diferentes edades en teleoperadores de una central de llamadas de la provincia de Málaga, siendo mayor la presencia de asimetría en sujetos de mayor edad.

- No parece existir relación entre la pérdida auditiva asimétrica con el resto de factores sociodemográficos, laborales y antecedentes médicos analizados en teleoperadores de una central de llamadas de la provincia de Málaga.

\section{CONFLICTO DE INTERESES}

Los autores declaran no tener ningún tipo de conflicto de intereses, ni ninguna relación económica, personal, política, interés financiero ni académico que pueda influir en su juicio; además de no haber recibido ningún tipo de beneficio monetario, bienes ni subsidios de alguna fuente que pudiera tener interés en los resultados de esta investigación.

\section{REFERENCIAS BIBLIOGRÁFICAS}

1. Asociación de Contac Center Española (ACE). Informe de Mercado 2017. [online]. 2017. [citado 03 Marzo 2018]. Disponible en la World Wide Web: http://www.ace.adigital.org/estudios/estudio_ace_2017.pdf

2. Vida Barea, Julián. Clasificación Nacional de Actividades Económicas (CNAE). [online]. 2009. [citado 04 Febrero 2018]. Disponible en la World Wide Web: https://www.cnae.com.es/index.php

3. Chouanière D, Boini S, Colin R. Conditions de travail et santé dans les centres d'appels téléphoniques. Documents pour le Medicin du Travail TF 191. [online]. 2011. [citado 14 Enero 2018], p.241-59. Disponible en la World Wide Web: www.inrs.fr/dms/inrs/CataloguePapier/DMT/TI-TF-191/tf191.pdf

4. Dossier INRS. Travail en centres d'appels téléphoniques. [online]. feb. 2011. [citado 14 Enero 2018], Disponible en la World Wide Web: http://2.238.129.10/cciaa/data/docs/INRS\%20dw58\%20call\%20center. pdf

5. Chatillon, J, Trompette, N. Observation de láctivité en CAT et limites d'exposition au bruit. Note documentaire ND 2314. Hygiène et sécurité au travail. [online]. sept. 2009, no. 216. [citado 14 Enero 2018], p.33-39. Disponible en la World Wide Web: www.inrs.fr/media.html?refINRS=ND\%202314

6. Cedric Aubert. Evaluation de la fatigue auditive peripherique et de ses determinants dans les centres d'appels telephoniques par le dispositif EchoScan R. Medecine humaine et pathologie. [online]. 2017. [citado 14 Enero 2018], Disponible en la World Wide Web: https://dumas.ccsd.cnrs.fr/dumas-01516132

7. Colaboradores de Wikipedia. Auricular [online]. 2018 [citado: 7 Marzo 2018], Disponible en la World Wide Web: https://es.wikipedia.org/w/index.php?title=Auricular\&oldid=105754406

8. Jacqueline A. Patel, Keith Broughton. Assessment of the noise exposure of call centre operators. Ann Occup Hyg 2002; 46(8): 653-61

9. Nicolas Trompette, Jacques Chatillon. Survey of noise exposure and background noise in call centers using headphones. J Occup Environ Hyg 2012; 9(6): 381-6

10. Pawlaczyk-Luszczynska, Malgorzata, Dudarewicz Adam, Zamojska-Daniszewska, Malgorzata, RutkowskaKaczmarek, Paulina, Zaborowski, Kamil. Noise exposure and hearing threshold levels in call center operators. 12th ICBEN Congress on Noise as a Public Health Problem. [online]. jun. 2017 [citado: 7 Marzo 2018], p. 1-11. Disponible en la World Wide Web: http://www.icben.org/2017/ICBEN\%202017\%20Papers/ SubjectArea01_Pawlaczyk-Luszczynska_0112_3664.pdf

11. Boletín Oficial del Estado. Real Decreto 286/2006, de 10 de marzo, sobre la protección de la salud y la seguridad de los trabajadores contra los riesgos relacionados con la exposición al ruido. [online]. marzo 2006, no. 60 [citado 04 Marzo 2018], p. 9842-48. Disponible en la World Wide Web: https://www.boe.es/ boe/dias/2006/03/11/pdfs/A09842-09848.pdf

12. Boletín Oficial de la Unión Europea. DIRECTIVA 2003/10/CE del Parlamento Europeo y del Consejo, de 6 de febrero de 2003, sobre las disposiciones mínimas de seguridad y de salud relativas a la exposición de los trabajadores a los riesgos derivados de los agentes físicos (ruido) (decimoséptima Directiva específica con arreglo al apartado 1 del artículo 16 de la Directiva 89/391/CEE). [online]. feb. 2003 [citado 04 Marzo 2018], p. 38-44. Disponible en la World Wide Web: https://www.boe.es/doue/2003/042/L0003800044.pdf

13. Ayse Coskun Beyan, Yucel Demiral, Arif Hikmet Cimrin, Alparslan Ergor. Call center and noise-induced hearingloss. Noise Health 2016; 18(81): 113-116 
14. A D'Alcamo, E Omeri, P Urso, S Fossati, M Coggiola, G Levizzani et al. Assessment of comfort and health status of Call Center'sworkers. G Ital Med Lav Ergon 2011; 33(3 Suppl): 348-50

15. Chia-Fen Chi, Yen-Hui Lin. An ergonomic evaluation of a call center performed by disabled agents. Percept Mot Skills 2008; 107(1): 55-64

16. Thomas Venet, Ayoub Bey, Pierre Campo, Joel Ducourneau, Quentin Mifsud, Charles Hoffmann et al. Auditory fatigue among call dispatchers working with headsets. Int J Occup Med Environ Health 2018; 31(2): $217-226$

17. Cid JM, Aguilar A. Cuestionario de Hábitos Cotidianos y Ruido Ambiental. En Guía de prácticas en psicopatología del lenguaje. 1997. Barcelona. Edicions Universitat de Barcelona

18. A Aguilar Alonso, J M $\mathbf{M}^{\mathrm{a}}$ Cid Rodríguez, E $\mathbf{M}^{\mathrm{a}}$ Aguilar Mediavilla. Ruido Ambiental y Capacidad Auditiva en Estudiantes Universitarios. Rev Logop Fon Audiol 2001; 21(4): 166-172

19. Eggermont, Jos J. Epidemiology and Genetics of Hearing Loss and Tinnitus. En Hearing Loss [online]. 2017 [citado 02 Julio 2018], p. 209-34. Disponible en la World Wide Web: 10.1016/B978-0-12-805398$0.00007-4$

20. KJ Cruickshanks, DM Nondahl, DS Dalton, ME Fischer, BE Klein, R Klein et al. Smoking, Central Adiposity and Poor Glycemic Control Increase Risk of Hearing Impairment. J Am Geriatr Soc 2015; 63(5): 918-24

21. ME Lutman, RRA Coles. Asymmetric sensorineural hearing thresholds in the non-noise-exposed UK population: a retrospective analysis. Clin. Otolaryngol 2009; 34: 316-321

22. Trung N Le, Louise V Straatman, Jane Lea, Brian Westerberg. Current insights in noise-induced hearing loss: a literature review of the underlying mechanism, pathophysiology, asymmetry, and management options. J Otolaryngol Head Neck Surg 2017; 46(1): 41

23. SV Fernandes, CM Fernandes. Medicolegal significance of asymmetrical hearing loss in cases of industria noise exposure. The Journal of Laryngology \& Otology 2010; 124: 105-55

24. David A Zapala, Robin E Criter, Jamie E Bogle, Larry B Lundy, Michael J Cevette, Christopher D Bauch. Pure-Tone Hearing Asymmetry: A Logistic Approach Modeling Age, Sex, and Noise Exposure History. J Am Acad Audiol 2012; 23: 553-570

25. Robert A Dobie. Does occupational noise cause asymmetric hearing loss?. Ear and hearing 2014; 35(5): $577-9$

26. Richard L Berg, William Pickett, James G Linneman, Douglas J Wood, Barbara Marlenga. Asymmetry in noise-induced hearing loss: Evaluation of two competing theories. Noise Health 2014;16:102-7

27. Xiaoxiao Wang, Nan Li, Lin Zeng, Liyuan Tao, Hua Zhang, Qiuling Yang et al. Asymmetric Hearing Loss in Chinese Workers Exposed to Complex Noise. Ear Hear 2016; 37(2): 189-193

28. ME Lutman, MA Cane, PA Smith. Comparison of manual and computer-controlled self-recorded audiometric methods for serial monitoring of hearing. Br J Audiol 1989; 23: 305-15

29. Lian Masterson, James Howard, Zi Wei Liu, John Phillips. Asymmetrical Hearing Loss in Cases of Industrial Noise Exposure: A Systematic Review of the Literature. Otol Neurotol 2016; 37: 998-1005

30. Jonathon P Whitton, Daniel B Polley. Evaluating the Perceptual and Pathophysiological Consequences of Auditory Deprivation in Early Postnatal Life: A Comparison of Basic and Clinical Studies. JARO: Journal of the Association for Research in Otolaryngology 2011; 12(5): 535-547

31. Alyson B Kaplan, Elliott D Kozin, Aaron Remenschneider, Kian Eftekhari, David H Jung, Daniel B Polley et al. Amblyaudia: Review of Pathophysiology, Clinical Presentation, and Treatment of a New Diagnosis. Otolaryngol Head Neck Surg 2016; 154(2): 247-55

32. Daniel B Polley, John H Thompson, Wei Guo. Brief hearing loss disrupts binaural integration during two early critical periods of auditory cortex development. Nature communications 2013; 4: 25-47

33. RS Vinodh, N Veeranna. Evaluation of acoustic shock induced early hearing loss with audiometer and distortion product optoacoustic emissions. Indian J Med Sci 2010; 64(3): 132-9

34. Trompette, Nicolas, Chatillon, Jacques. Evaluation des risques pour l'audition des opérateurs des centres d'appels téléphoniques et solutions de prevention. Notes scientifiques et Techniques d'INRS NS 289. [online]. dic. 2012 [citado 10 Octubre 2018], p. 49. Disponible en la World Wide Web: www.inrs.fr/dms/ inrs/Publication/C-1-1-059...127.../ns289.pdf

35. Elizabeth Francis Beach, Warwik Williams, Megan Gilliver. The objective subjective assessment of noise: Young adults can estimate loudness of events and lifestyle noise. International Journal of Audiology 2012; 51: 444-9

36. Cory DF Portnuff, Brian J Fligor, KathrynArehart. Self-report and long-term field measures of MP3 player use: How accurate is self-report? International Journal of Audiology 2013; 52: 33-40 